\title{
Krankheiten mit labilem Dispersionsgrad der Säfte.
}

\author{
Von
}

\section{R. Geigel.}

(Eingegangen am 21. März 1922.)

Es gibt eine kleine Anzahl von Krankheiten der Atmungsorgane, mit denen die Pathologie noch nichts Rechtes anzufangen weiß. Gemeinschaftlich ist ihnen die Atemnot, die sie, oft in einzelnen Anfällen, begleitet. Das ist aber nur ein äuBerliches Merkmal. Ich werde zeigen, daß sie auch innerlich und ihrem Wesen nach miteinander verwandt sind. $\mathrm{Zu}$ diesem Zweck und da ich die klinischen Erscheinungen der einzelnen Krankheitsbilder als bekannt voraussetzen kann, will ich davon nur das hervorheben, worin ihre Verwandtschaft begründet zu sein scheint.

1. Das Asthma bronchiale. Den asthmatischen Anfall kennt jeder. Mechanisch wird er wohl am besten nach der Weise von Biermer erklärt, wonach es sich um einen Krampf der Bronchialmuskeln handelt. Die Atemnot, der Tiefstand des Zwerchfells, die Lungenblähung, der Schachtelton, das alles ist nur die Folge des Muskelkrampfs, der die Luft wohl in die Lungen eindringen läßt, aber nur schwer wieder heraus. Das rasche Kommen und Gehen des Anfalls, die Tatsache, daß der Anfall reflektorisch ausgelöst werden kann, allgemein nervöse Momente mitspielen, woher die Krankheit auch den Namen Asthma nervosum bekommen hat, ist wohl damit vereinbar.

Eines ist aber damit nicht erklärt, die Beschaffenheit des Sekrets in den Bronchien und das, was man im Auswurf findet, die Curschmannschen Spiralen, die Charcot-Leydenschen Krystalle, die eosinophilen Zellen. Im Anfang ist das Sputum außerordentlich zäh, spärlich und wenn es dünner wird und an Menge zunimmt, dann ist die Gewalt des Anfalls schon gebrochen.

Betrachten wir den Vorgang vom kolloidchemischen Standpunkt aus, so unterliegt es keinem Zweifel, daß die zwei wohlbekannten Vorgänge: die Koagulation und die Peptisation hier ihre Rolle spielen. Innerhalb gewisser Grenzen wird bei der Koagulation der Dispersionsgrad eines kolloiden Systems vermindert, bei der Peptisation erhöht. Dal das Bronchialsekret ein kolloides System, eine kolloide Lösung in Wasser, ein Sol ist, darüber ist nicht zu streiten. Schon bei der Bildung und Aus: scheidung des Bronchialschleims tritt uns der Vorgang der Peptisation 
entgegen. Man kann es ja in den Becherzellen und den Drüsenzellen direkt sehen, wie der Inhalt der Zellen die Körnung verliert, dünner flüssig wird und so zur Ausscheidung kommen kann. Das Eigentümliche beim asthmatischen Anfall besteht nur darin, daß im ergossenen Sekret sogleich eine Vermehrung der Zähigkeit eintritt, also ein Zeichen verminderter Dispersion und daß man diesen Vorgang wieder mit dem Mikroskop verfolgen kann. Wenn der Dispersionsgrad in einem kolloiden System vermindert wird, so ändern sich manche physikalische Eigenschaften, elektrische, Lichtbrechungsvermögen und andere; von der größten Wichtigkeit aber ist die Veränderung des Reibungskoeffizienten, der Zähigkeit, der Art, daß die Änderung der Zähigkeit geradezu als der Maßstab für den Dispersionsgrad angesehen wird. Bei der Verminderung des Dispersionsgrads kommt es je nach der Größe der Änderung zur Koagulation und zur Ausflockung. Und das kann man wieder im Auswurf beim asthmatischen Anfall sehen. Aus dem anscheinend homogenen "Schleim" scheiden sich geformte Teile aus: die Curschmannschen Spiralen in Form von gedrehten Fäden. Sie bestehen z. T. aus Fibrin, z. T. aus Mucin, beide waren in kolloidaler Lösung und scheiden sich jetzt in fester Formart wie so oft als Fäden aus, wenn der Dispersionsgrad sich um ein gewisses Maß vermindert. Die spiralige Drehung der Fäden ist ein sekundärer Vorgang beim Verschieben der Gebilde in den Luftwegen.

Aus kolloiden Lösungen fällt die disperse Phase allermeist amorph aus, aus molecularen und iondispersen Lösungen krystallinisch. Aber wie der Übergang von Kolloiddispers und Soldispers fließend ist, so ist auch der Unterschied zwischen amorph und krystallinisch kein sprungartiger und Zwischenformen kommen vor. So ist es auch mit den Charcot-Leydenschen Krystallen. Man will an ihnen eine schwache Doppelbrechung beobachtet haben. Ist das richtig, so könnten es Pyramiden nach dem hexagonalen System sein. An solchen Gebilden, allerdings nicht beim Asthma, habe ich keine Spur von Doppelbrechung nachweisen können. Sie sehen auch mehr wie Zwischenformen zwischen Krystall und Amorphem aus. Auch die Krystalle, die ächten, haben, wenn sie sich bilden, zuerst nur die stumpfen, gerundeten Kanten, die man auch an den Charcot-Leydenschen Pyramiden sieht. Im ganzen sind auch sie nichts Anderes, als die fest gewordene disperse Phase eines kolloiden Systems.

Eine besondere Wichtigkeit schreibe ich den eosinophilen Zellen zu. Die Curschmannsehen Spiralen entstehen zwar wahrscheinlich nicht daraus, aber beide kommen fast stets zusammen vor. Bedeutsamerweise finden sich die eosinophilen Zellen beim Asthma bronchiale auch im Blut vermehrt, also handelt es sich gewiß nicht um eine Bildung im Bronchialsekret an Ort und Stelle. Genug, in Blut haben viele Leuko 
cyten, mehr als gewöhnlich eine grobe Körnung ihres Inhalts erfahren. Denn das ist das Bezeichnende für die eosinophilen Zellen, die man auch ohne Färbung an ihren groben Körnern erkennen kann, nach der Färbung mit sauren Farbstoffen wie Eosin freilich viel leichter. Die Eigenschaft der groben Körner, sich mit Eosin zu färben, geht uns hier nichts weiter an, das ist eine sekundäre Sache, die Hauptsache ist die grobe Körnung des Protoplasmas. Und die ist wieder, kolloidchemisch gesprochen, ein Vorgang der Koagulation, also der Verminderung des Dispersionsgrades. Daß die Eosinophilie nicht bloß eine gleichgültige Begleiterscheinung sein kann, dafür spricht manches. Erstens die RegelmäBigkeit, mit der sie auftritt, ferner die Beobachtung, daß sie nicht nur den Anfall überdauert, sondern ihm auch vorangehen kann oder schließlich als einzige Erscheinung der Krankheit, auch ohne Anfälle, auftreten, besonders bei Kindern.

Denn der asthmatische Anfall ist nicht die Krankheit, er ist nur der Ausdruck dafür, daß der Kranke ein Leiden hat, auf dessen Boden sich die Anfälle entwickeln. Das Kommen und Gehen der Anfälle hat etwas Rätselhaftes, um nicht zu sagen Bizarres an sich. Und über die Annahme einer besonderen Konstitution oder Diathese oder wie man es sonst heißen will, kommt man beim Asthma nicht hinaus. Die erbliche Anlage spielt dabei sicher eine wichtige Rolle, darin sind sich alle einig.

Wie der Anfall gekommen ist, so vergeht er wieder ,,von selbst", wie man sagen möchte. Dem liegt aber jetzt das Gegenstück zugrunde von dem, was zum Anfall geführt hat. Das außerordentlich zähe Sputum wird dünnflüssig, kann infolgedessen viel leichter ausgeworfen werden. Und sowie der Auswurf dünn wird, nimmt die Atemnot rasch ab und der Anfall ist in kurzer Zeit zu Ende. Nach den Begriffen der Kolloidchemie ist jetzt eine Erhöhung des Dispersionsgrades eingetreten: die Peptisation. Man weiß, daß die Peptisation durch mancherlei Einflüsse, oft recht geringe, in kolloiden Systemen angeregt werden kann, so namentlich auch durch Fermente. Solche entstehen z. B. durch Lebenseigenschaften und den Zerfall von weißen Blutkörperchen oft genug. Die Einschmelzung der Gewebe bei der Eiterung beruht darauf. Ob auch beim Abklingen des asthmatischen Anfalls die weißen Blutzellen diese Rolle spielen, was möglich wäre, weiß ich nicht. Jedenfalls, das wollen wir festhalten, liegen dem Asthma bronchiale zwei entgegengesetzte Vorgänge aus der Kolloidchemie zugrunde, eine Verminderung und dann eine Erhöhung des Dispersionsgrades.

Therapeutisch ist das Jodkalium von Bedeutung.

2. Der eosinophile Katarrh, zuerst beschrieben von F. A. Hoffmann und seinem Schüler Teichmüller, ist vielleicht nichts anderes als eine leichtere Form von Bronchialastbma mit Zurücktreten der Anfälle. Die Krankheit verläuft mehr wie ein heftiger und sehr hartnäckiger 
Katarrh. Der Schleim ist nicht ganz so zäh wie beim Asthma, enthält aber auch viele eosinophile Zellen, gelbliche Klumpen, in denen sie sich massenhaft finden, auch Charcot-Leyden sche Krystalle und hier und da auch Curschmannsche Spiralen. Die Atemnot ist mäBig, der Husten aber mühsam und quälend. Im Blut sind die Eosinophilen vermehrt. Das Hauptarzneimittel ist wieder Jodkalium.

Dem „Katarrh sec" von Laënnec gestehe ich eine Sonderstellung nicht zu, er ist wohl nichts anderes als eine Abart der gewöhnlichen Bronchitis sicca.

3. Der pituitöse Katarrh ist keine Entzündung, nur eine Ausscheidung einer dünnen Lösung, besser gesagt, eines Hydrosols, denn sie hat alle Eigenschaften einer kolloidalen Lösung. Das Sekret bildet beim Ausgießen keine Tropfen, sondern einen ununterbrochenen Strahl, wenn man das Sputum auch nicht zäh nennen kann im Vergleich mit dem Auswurf beim Asthma oder der Bronchitis plastica, so ist die Kohäsion doch bedeutender als beim Wasser, das spezifische Gewicht ist klein, der Eiweißgehalt gering. Der Auswurf steigert sich anfallsweise, ist aber auch in der $Z$ wischenzeit dünn und reichlich. Er enthält eosinophile Zellen in nicht unbeträchtlichen Mengen, auch Curschmannsche Spiralen und Charcot-Leyden sche Krystalle kommen vor.

Die Krankheit trägt ihren alten Namen „Asthma humidum“ nicht mit Unrecht. Im Anfall ist die Atemnot stark, er gleicht überhaupt dem asthmatischen Anfall sehr, manchmal auch einem Anfall von Lungenödem. Auch im Blut findet sich Eosinophilie. Jodkalium ist auch hier wieder ein Hauptmittel.

Die Beschaffenheit des Auswurfs ist der beim Asthma vollständig entgegengesetzt. Ob aber dem dünnen Auswurf nicht die kurzdauernde Bildung eines zähen vorausgegangen, wäre noch zu untersuchen. Ausgeschlossen ist das nicht, die Atemnot spricht dafür. Diese ist anders als beim Lungenödem und ganz so wie beim Asthma. Höchstwahrscheinlich besteht wie beim Asthma auch zunächst Koagulation, die aber so rasch vorübergeht, daß sie nicht auffällt und durch den Vorgang der Peptisation, die sogleich einsetzt, wird das dünne Sputum geliefert.

4. Das Heuasthma verläuft gerade so wie das Asthma bronchiale mit allen seinen Begleiterscheinungen, der Koagulation, der Eosinophilie, der nachfolgenden Peptisation, aber hier kennen wir wenigstens die Ursache im Einatmen von Pollen gewisser Gramineen, gegen die die Atmungsorgane mancher Leute eine rätsellhafte Empfindlichkeit zeigen. Diese Überempfindlichkeit ist offenbar in einer erblichen Anlage begründet. In der Familie der Kranken kommt Heuasthma oder eine der genannten anderen Formen der Gruppe vor oder etwas ähnliches, wie nasale Hydrorrhöe oder exsudative Diathese oder Enteritis membranacea oder dergleichen, was auf Labilität des Dispersionsgrades hinweist und 
was man bis jetzt mangels näherer Einsicht unter dem Namen einer Konstitutionsanomalie zusammenfaßt.

Immerhin, beim Heuasthma kennt man die Krankbeitsursache, es sind die in den Blütenpollen enthaltenen Stoffe und darauf hat man ja bekanntlich auch die spezifische Therapie mit dem Pollantin gegründet. Warum aber das Gift der Pollen nicht auf alle Menschen gleicb einwirkt und nur auf eine bestimmte kleine Zahl, davon weiß man garnichts. Eine gewisse und zwar erbliche Anlage ist also auch hier notwendig, damit einer zur Zeit der Heuernte sein Heuastbma, ein anderer seinen Heuschnupfen bekommt. Übrigens ist das Jodkalium auch beim Heuasthma als wirksames Mittel in Gebrauch.

Hiermit bätten wir die Formen zusammengestellt, die meines Erachtens ihrem Wesen nach zusammen gehören. Warum, wird man fragen, sind die croupöse Pneumonie und die Diphtherie nicht dabei? Bei der croupösen Pneumonie ist der Vorgang der Koagulation, und noch dazu nach gesetzmäßiger Frist, die Peptisation für die Krankheit bezeichnend, die Hepatisation, ein Vorgang verminderten, die Lösung erhöhten Dispersionsgrades. Und bei der Diphtherie beruht die Gefahr der Krankbeit gerade auf der bezeichnenden Koagulation, wodurch die diphtherischen Exsudate und die Croupmembranen gebildet werden, die leider vergebens auf den Vorgang der Peptisation warten. Gewiß ist der Vorgang der Koagulation und bei der croupösen Pneumonie auch der Peptisation eine Folge der Lebenstätigkeit uns wohlbekannter Bakterien aber, und das ist der Grund, warum ich diese Krankheiten der hier besprochenen Gruppe nicht einreihe, sie wirken auf alle Menschen gleich oder ziemlich gleichartig ein und die Konstitution und vor allem auch die erbliche Anlage spielt da gar keine Rolle. Das ist der große Gegensatz zum Astbma und den verwandten Formen. Bei der Bronchitis plastica acuta und chronica könnte man zweifelhaft sein, ob sie durch eine Infektion hervorgerufen werden. Sollte das nicht zutreffen, so wären sie hier anzuschließen.

Ủberblicken wir nun die ganze Gruppe von Krankheiten, die wir hier kurz zusammengefaßt haben, und sehen nach, was ihnen gemeinsam ist, so treffen wir zunächst auf einige Eigenschaften, die sie insgesamt nicht haben. Von keiner kann man sagen, daß eine Infektion irgend wahrscheinlich ist. Auch ein ganz bestimmter Reiz, der die Luftwege betroffen hätte, ist außer beim Heuasthma nicht anzuschuldigen und auch hier ist es einer, der tausend andere Menschen alljährlich zur gleichen Zeit angreift, ohne zum Heuasthma zu fübren. Dagegen spielt die erbliche Anlage bei allen Formen eine ungemein wichtige Rolle, die vererbte Empfindlichkeit, durch die eine kleine Minderzahl gegen, jedenfalls weit verbreitete, Schädlichkeiten ausgezeichnet ist und vor allem die vererbte Art, wie der Körper auf die eingetretene Schädigung antwortet. Nicht 
die spezifische Infektion wie bei der croupösen Pneumonie oder bei der Diphtherie bewirkt den bezeichnenden Vorgang der Koagulation mit vielleicht folgender Peptisation, sondern das macht bei unserer Gruppe die Gegenwirkung des Körpers ganz allein. Und daß er so auf eine meist gar nicht spezifische Ursache antwortet, das liegt in seiner Natur, in seiner erblichen Anlage, mit einem Wort, in seiner Konstitution begründet.

Kolloide Systeme sind, wie man weiß, im ganzen sehr labil, sie seien in einem fort in Unruhe, bat man nicht mit Unrecht gesagt. Darauf beruht die Fähigkeit kolloider Lösungen, Träger des Lebens zu werden, in erster Linie. Nur so ist es möglich, daß unter Beibehaltung der äußeren Form eine ungeheuer große Beweglichkeit bald mehr, bald weniger eintretend, nach Bedarf wechselnd sich geltend macht, wie man es bei den Lebensvorgängen auf Schritt und Tritt wahrnimmt.

Aber auch hier besteht offenbar ein Unterschied wenigstens dem Grade nach und dieser Unterschied vererbt sich wie tausend andere Dinge bis in die kleinsten Einzelheiten hinein, zum Erschrecken förmlich, worüber sich als über längst Bekanntes kein Mensch mehr wundert.

Wahrscheinlich beruht die ganze Konstitution auf der Beschaffenheit der kolloiden Systeme, aus denen der Körper zusammengesetzt ist und die Träger des Lebens sind. Damit ist nicht nur das gemeint, was man die „Säfte" des Körpers zu nennen pflegt, auch das Protoplasma der Zellen ist ein Sol, unter Umständen ein Gel. Aber bei der vorliegenden Krankheitsgruppe ist das besondere Verhalten der kolloiden Systeme mit den Händen zu greifen. Sie zeichnen sich, offenbar vererbt, durch eine besondere Labilität des Dispersionsgrades aus. Überaus leicht führt das zur Koagulation und ïberaus leicht wieder zur Peptisation. Zeitlich ist bei der einen Form der eine, bei der anderen der andere Vorgang ausgedehnter. Noch mehr, die Labilität scheint sich nicht auf das, was man "Säfte" nennt, zu beschränken, wie die Erscheinung der Eosinophilie lehrt, die allen Formen der Gruppe gemeinsam ist.

Die Vermehrung der eosinophilen Zellen ist doch ganz merkwürdig. Sie fehlt bei keiner Form der Gruppe. Man kann nicht sagen, daß sie an der veränderten Schleimhaut entstanden sind, oder sich dort nur angelockt aus dem Blut finden. Dann müBte das Blut ärmer daran geworden sein, es ist aber, wie wir wissen, reicher daran. Freilich ist die Bildung eosinophiler Zellen aus neutrophilen oder basophilen meines Wissens noch nie direkt beobachtet worden und wenn ich mir vorstelle, daß beim Asthma, dem eosinophilen Katarrh und den anderen Formen weiße Blutzellen eosinophil werden, $d . \mathbf{h}$. in ihrem Protoplasma eine gröbere Körnung annehmen, so ist das freilich vorläufig noch eine Hypothese, für die der Beweis noch mangelt. Aber einiges könnte ich doch für meine Meinung anführen, namentlich dafür, daß die eosinophilen 
Zellen beim Asthma nicht aus dem Blut, wo sie sich schon vorher befanden, ausgeschieden werden. Die eosinophilen Zellen im Auswurf beim Asthma sind nämlich wie man schon weiß, einkernig und die eosinophilen Zellen im Blut sind vielkernig. Wenn die eosinophilen Zellen immer neu als solche gebildet würden, so wäre ihr rasches Auftreten bei den Krankheiten dieser Gruppe doch recht merkwürdig.

Immer tritt Atemnot auf und hält an zur Zeit, wo die Koagulation vorherrscht, und sie vergeht mit dem Eintritt der Peptisation. Vom Jodkalium weiß man, daß es den Dispersionsgrad erhöht, es müßte also, vom kolloidchemischen Standpunkt aus betrachtet, günstig wirken und das tut es auch wirklich bei allen.

Aus alledem leite ich für mich die Berechtigung ab, die aufgezähiten Krankheitsformen ihrem Wesen nach in eine Gruppe zu bringen und ihnen die Eigenschaft zuzuschreiben, daß der Dispersionsgrad der kolloidalen Lösungen sich in einem besonders labilen Zustand befindet. Ursachen, die sich unserer Kenntnis entziehen, vielleicht weil sie zu unscheinbar sind, genügen, um den Vorgang der Koagulation und dann wieder der Peptisation in Gang zu bringen, also den Grad der Dispersion zu vermindern und dann wieder zu erhöhen. Die Erscheinung der Eosinophilie weist nach meiner Deutung mit Sicherheit darauf hin, daß diese Labilität sich nicht nur auf einen kleinen Raum beschränkt, sondern eine gewisse allgemeine Verbreitung bedeutet.

Dabei bin ich mir dessen wohl bewußt, daß diese Art der Konstitutionsanomalie sich nicht nur in Erkrankungen der Bronchien und der Lungen äußert. Gewiß entstehen auch andere Krankheitsformen auf dem gleichen Boden. Ich erinnere hier nur an die nasale Hydrorrhöe, an die Enteritis membranacea, an die Arthritis deformans. Wahrscheinlich spielt die gute oder schlechte Wirksamkeit der endokrinen Drüsen auch eine wiohtige Rolle und als an ein Beispiel davon denke ich an das Myxödem.

So wäre bier noch manches zu sagen und ich hoffe bestimmt, daß mit dem weiteren Ausbau der Kolloidchemie und deren Anwendung auf die Pathologie noch manches bisher Dunkle besser aufgeklärt werden wird. 